\title{
Paradox of soft singularity crossing and its resolution by distributional cosmological quantities
}

\author{
Zoltán Keresztes, ${ }^{1,2, *}$ László Á. Gergely, ${ }^{1,2, \dagger}$ and Alexander Yu. Kamenshchik ${ }^{3,4, \ddagger}$ \\ ${ }^{1}$ Department of Theoretical Physics, University of Szeged, Tisza Lajos krt 84-86, Szeged 6720, Hungary \\ ${ }^{2}$ Department of Experimental Physics, University of Szeged, Dóm Tér 9, Szeged 6720, Hungary \\ ${ }^{3}$ Dipartimento di Fisica and INFN, via Irnerio 46, 40126 Bologna, Italy \\ ${ }^{4}$ L. D. Landau Institute for Theoretical Physics, Russian Academy of Sciences, Kosygin Street 2, 119334 Moscow, Russia
}

(Received 5 April 2012; published 20 September 2012)

A cosmological model of a flat Friedmann universe filled with a mixture of anti-Chaplygin gas and dustlike matter exhibits a future soft singularity, where the pressure of the anti-Chaplygin gas diverges (while its energy density is finite). Despite infinite tidal forces the geodesics pass through the singularity. Because of the dust component, the Hubble parameter has a nonzero value at the encounter with the singularity, therefore the dust implies further expansion. With continued expansion however, the energy density and the pressure of the anti-Chaplygin gas would become ill-defined hence from the point of view of the anti-Chaplygin gas only a contraction is allowed. Paradoxically, the universe in this cosmological model would have to expand and contract simultaneously. This obviously could not happen. We solve the paradox by redefining the anti-Chaplygin gas in a distributional sense. Then a contraction could follow the expansion phase at the singularity at the price of a jump in the Hubble parameter. Although such an abrupt change is not common in any cosmological evolution, we explicitly show that the set of Friedmann, Raychaudhuri and continuity equations are all obeyed both at the singularity and in its vicinity. We also prove that the Israel junction conditions are obeyed through the singular spatial hypersurface. In particular we enounce and prove a more general form of the Lanczos equation.

\section{INTRODUCTION}

The problem of cosmological singularities has been attracting the attention of theoreticians working in gravity and cosmology since the early fifties [1-3]. In the sixties general theorems about the conditions for the appearance of singularities were proven $[4,5]$ and the oscillatory regime of approaching the singularity [6], the Mixmaster universe [7] was discovered. Basically, until the end of nineties almost all discussions about singularities were devoted to the big bang and big crunch singularities, which are characterized by a vanishing cosmological radius.

However, kinematical investigations of Friedmann cosmologies have raised the possibility of sudden future singularity occurrence [8-18], characterized by a diverging $\ddot{a}$ whereas both the scale factor $a$ and $\dot{a}$ are finite. Then, the Hubble parameter $H=\dot{a} / a$ and the energy density $\rho$ are also finite, while the first derivative of the Hubble parameter and the pressure $p$ diverge. Until recent years, however, sudden future singularities attracted only a limited interest among researchers. The interest grew due to two reasons. The recent discovery of the cosmic acceleration [19] has stimulated the elaboration of dark energy models, responsible for such a phenomenon (see e.g., for review [20]). Remarkably in some of these models the sudden singularities arise quite naturally. Another source for the interest in

\footnotetext{
*zkeresztes@titan.physx.u-szeged.hu

†ergely@physx.u-szeged.hu

Alexander.Kamenshchik@bo.infn.it
}

sudden singularities is the development of brane models $[10,11,18]$, where singularities of this kind could arise naturally (sometimes these singularities, arising in braneworld models, are called "quiescent" [10]).

In the investigations devoted to sudden singularities one can distinguish three main topics. The first of them deals with the question of the compatibility of the models possessing soft singularities with observational data $[15,21,22]$. The second direction is connected with the study of quantum effects [11,17,23-25]. Here one can see two subdirections: the study of quantum corrections to the effective Friedmann equation, which can eliminate classical singularities or at least, change their form $[10,17,23]$; and the study of solutions of the WheelerDeWitt equation for the quantum state of the universe in the presence of sudden singularities [24,25]. The third direction is connected with the possibility of the sudden singularity crossing in classical cosmology [25-29]. The present paper is devoted exactly to this topic.

A particular feature of the sudden future singularities is their softness [26]. As the Christoffel symbols depend only on the first derivative of the scale factor, they are regular at these singularities. Hence, the geodesics are well behaved and they can cross the singularity [26]. One can argue that the particles crossing the singularity will generate the geometry of the spacetime, providing in such a way a "soft rebirth" of the universe after the singularity crossing [29]. Note that the possibility of crossing of some kind of cosmological singularities was noticed already in the early paper by Tipler [30]. A close idea of integrable 
singularities in black holes, which can give origin to a cosmogenesis was recently put forward in Ref. [31].

As a starting point we consider an interesting example of a sudden future singularity - the big brake which was discovered in Ref. [32] while studying a particular tachyon cosmological model. The particularity of the big brake singularity consists in the fact that the time derivative of the scale factor is not only finite but exactly equal to zero. That makes the analysis of the behavior in the vicinity of singularity especially convenient. In particular, in Ref. [22] it was shown that the predictions of the future of the universe in this model [32] are compatible with the supernovae-type Ia data, while in Refs. [24,25] some quantum cosmological questions were studied in the presence of the big brake singularity.

The simplest cosmological model allowing a big brake singularity was also introduced in Ref. [32]. This model is based on the perfect fluid, dubbed "anti-Chaplygin gas". This fluid is characterized by the equation of state

$$
p=\frac{A}{\rho},
$$

where $A$ is a positive constant. Such an equation of state arises, for example, in the theory of wiggly strings [33]. In paper [32] a fluid obeying the equation of state (1) was called "anti-Chaplygin gas" in analogy with the Chaplygin gas [34] which has the equation of state $p=$ $-A / \rho$ and has acquired some popularity as a candidate for a unified theory of dark energy and dark matter [35].

An explicit example of the crossing of the big brake singularity was described in detail in paper [29], in which the tachyon model $[22,32]$ was investigated. In this model the tachyon field passes through the singularity, continuing its evolution with a recollapse towards a big crunch. In a simpler model, based on the anti-Chaplygin gas, such a crossing is even easier to understand.

The next natural step in the analysis of the soft singularities seems to be obvious. One can consider a soft singularity of more general type than the big brake by adding to the tachyon matter or to the anti-Chaplygin gas some dustlike matter. However, in this case the traversability of the singularity seems to be obstructed. The main reason for this is that while the energy density of the tachyonic field (or of the anti-Chaplygin gas) vanishes at the singularity, the energy density of the matter component does not, leaving the Hubble parameter at the singularity with a finite value. Then some kind of paradox arises: if the universe continues its expansion, and if the equation of state of the component of matter, responsible for the appearance of the soft singularity (in the simplest case, the anti-Chaplygin gas) is unchanged, then the expression for the energy density of this component becomes imaginary, which is unacceptable. The situation looks rather strange: indeed, the model, including dust should be in some sense more regular than a single exotic fluid, the anti-Chaplygin gas. Thus, if the model based on the pure anti-Chaplygin gas has a traversable big brake singularity, than the more general singularity arising in the model based on the mixture of the anti-Chaplygin gas and dust should also be traversable.

Related to that, it was recently shown that general soft singularities arising in the Friedmann model, filled with the scalar field with a negative potential, inversely proportional to this field are traversable. So, what could be wrong with the simple two-fluid model? One can see that what we face is some sort of a clash between the equation of state of one of these fluids and the dynamics (the Friedmann and Raychaudhuri equations) and energy conservation equations. In this paper we shall try to resolve this paradox, insisting on the preservation of the equation of state of the anti-Chaplygin gas. The price which one has to pay for it is the obligatory use of the generalized functions for some cosmological quantities. Namely, the anti-Chaplygin gas remains physical if rather a recollapse follows, but then the Hubble parameter would have a sharp jump, obstructing the validity of the Raychaudhuri equation (the second Friedmann equation) in the usual sense of functions. Thus, apparently, the evolution cannot be continued through the soft singularity, unless treating the cosmological quantities as distributions. We claim that such a generalization is mathematically rigorous, moreover, the introduction of distributions is not so drastic, as it looks at the first sight, as the pressure of the anti-Chaplygin gas diverges anyhow at the soft singularity (as it does for the tachyon field). Then in the Conclusion we shall dwell on the possible physical sense of the proposed constructions and its possible alternatives.

The plan of the paper is as follows. In Sec. II we discuss generic Friedmann spacetimes, which admit $\dot{H}=-\infty$ type singularities, while the Hubble parameter $H$ remains finite. Such singularities are related to corresponding divergencies in the pressure of the perfect fluid filling the Friedmann universe (while its energy density stays finite). We investigate the kinematics, the geodesic equations, the geodesic deviation equations in the vicinity of these singularities and also prove that these singularities are weak.

In Sec. III we discuss a mixture of the anti-Chaplygin gas and dust in a flat Friedmann universe and explain the essence of the paradox. We explicitly derive the behavior of the energy density and pressure in the vicinity of the soft singularity and we solve the geodesic equations in this region. While the singularity turns to be traversable by the geodesics, the explicit solution also shows that the Raychaudhuri equation is violated at the singularity.

In Sec. IV we add generalized distributional contributions to both the pressure and energy density, such that (a) the equation of state of the anti-Chaplygin gas still holds and (b) the singularity becomes traversable. We also perform checks of the Friedmann, Raychaudhuri and continuity equations, which all hold valid across the 
singularity in a distributional sense. In the process we employ a number of propositions on distributions presented and proved in Appendix A. For the convenience of the reader we present a related semiheuristic discussion of two known distributional identities in Appendix B. We stress that the distributional modifications of the energy density and pressure do not modify the cosmological evolution, but they make possible the soft singularity crossing.

In Sec. $\mathrm{V}$ we revisit the junction conditions along a spacelike hypersurface in a flat Friedmann universe. The future soft singularity represents such a spatial hypersurface, along which the energy-momentum tensor diverges. Extending the spacetime through this hypersurface is possible by obeying both Israel junction conditions. While the first condition, requiring the continuity of the induced metric is easy to satisfy (the metric stays regular at the soft singularity), the second condition relates the jump in the extrinsic curvature to the distributional part of the energy-momentum tensor through the Lanczos equation. We will show that in flat Friedmann spacetimes the Lanczos equation holds for a more general class of distributional energy-momentum tensors. With this we give a second proof that the generalized distributional energymomentum tensor assures the traversability of the soft singularity. In the process we employ a simple form of the Lanczos equation valid in flat Friedmann universes, derived in Appendix C.

We summarize our results and give some further outlook in the concluding remarks.

We chose $c=1$ and $8 \pi G / 3=1$. A subscript $S$ denotes the value of the respective quantity at the soft singularity.

\section{PRESSURE SINGULARITIES IN FLAT FRIEDMANN UNIVERSES}

The line element squared of a flat Friedmann universe can be written as

$$
d s^{2}=-d t^{2}+a^{2}(t) \sum_{\alpha}\left(d x^{\alpha}\right)^{2},
$$

where $x^{\alpha}(\alpha=1,2,3)$ are Cartesian coordinates. The evolution of the Friedmann universe is governed by the Raychaudhuri (second Friedmann) equation

$$
\dot{H}=-\frac{3}{2}(\rho+p)
$$

and by the continuity equation for the fluid

$$
\dot{\rho}+3 H(\rho+p)=0 .
$$

Here the dot denotes the derivative with respect to cosmological time $t$. A first integral of this system is given by the first Friedmann equation

$$
H^{2}=\rho .
$$

It is easy to see that the Raychaudhuri equation can be obtained from the first Friedmann and the continuity equations.

\section{A. Kinematics in the vicinity of sudden singularities}

Sudden singularities are characterized by finite $H$ and $\dot{H} \rightarrow-\infty$ (finite $\dot{a}$ and $\ddot{a} \rightarrow-\infty$ ) at some finite scale factor $a$. The energy density of the fluid is finite but its pressure diverges at this type of singularity, therefore the term "pressure singularity" is also used. Then, we would like to emphasize the fact that there is an essential difference between the sudden singularities with $H=0$ and with $H>0$. As already mentioned in the Introduction, in the first case, which is called big brake, the universe begins contracting and running towards the big crunch singularity. This occurs exactly in models based on tachyon field with a particular potential $[22,29,32]$ or in the anti-Chaplygin gas models. In the case of the model based on the mixture of one of this fluids and dust, we encounter the second situation when the value of the Hubble constant is positive at the moment of encounter with the sudden singularity. That means that after crossing the singularity the universe should continue its expansion, but the anti-Chaplygin gas becomes ill-defined (shown in detail in Sec. III), devoted to the model based on the mixture of the anti-Chaplygin gas and dust.

One possible way of overcoming this obstacle is to allow the jump in the sign of the Hubble parameter, which as mentioned in the Introduction leaves valid the first Friedmann equation, the continuity equation and the equation of state, while making invalid the Raychaudhuri equation. This last obstacle can be cured by the accepting the distributional Dirac $\delta$-function-type contributions into the pressure and the energy density, which is described in detail in the Sec. IV.

\section{B. Geodesics in the vicinity of sudden singularities}

The geodesic equations in flat Friedmann space-time are

$$
\begin{aligned}
& \frac{d^{2} x^{\alpha}}{d \lambda^{2}}+2 \frac{\dot{a}}{a} \frac{d t}{d \lambda} \frac{d x^{\alpha}}{d \lambda}=0, \\
& \frac{d^{2} t}{d \lambda^{2}}+a \dot{a} \sum_{\alpha}\left(\frac{d x^{\alpha}}{d \lambda}\right)^{2}=0,
\end{aligned}
$$

where $\lambda$ is an affine parameter. Integrating these equations yields

$$
\begin{gathered}
\frac{d x^{\alpha}}{d \lambda}=\frac{P^{\alpha}}{a^{2}}, \\
\left(\frac{d t}{d \lambda}\right)^{2}=\epsilon+\frac{P^{2}}{a^{2}},
\end{gathered}
$$

with $P^{\alpha}, \epsilon$ integration constants and $P^{2}=\sum_{\alpha}\left(P^{\alpha}\right)^{2}$. The quantity $\epsilon$ is fixed by the length of the tangent vector $u^{a}$ of 
the geodesic as $\epsilon=-u_{a} u^{a}$; i.e., one for timelike and zero for lightlike orbits. In a comoving system $P^{\alpha}=0$ and $t=\lambda$ is affine parameter.

Equations (8) and (9) are singular only for vanishing scale factor (see also Ref. [26]). Therefore, the existence of a solution $t(\lambda), x^{\alpha}(\lambda)$ of Eqs. (8) and (9) is assured by the Cauchy-Peano theorem for any nonzero $a$ (including the soft singularity). Thus the functions $t(\lambda)$ and $x^{\alpha}(\lambda)$, i.e., the geodesics can be continued through the singularity occurring at finite scale factor. Only derivatives of higher order than two of $t(\lambda)$ and $x^{\alpha}(\lambda)$ are singular (as they contain $\ddot{a})$, however these do not appear in the geodesic equations. Pointlike particles moving on geodesics do not experience any singularity. Thus, as we argued in the preceding paper [29] one is not obliged to consider such a singularity as a final state of the universe. Indeed, passing through this singularity the matter recreates also the spacetime in a unique way, at least for such simple models, as those based on Friedmann metrics.

\section{Deviation equation in the vicinity of sudden singularities}

The 3 -spaces with $t=$ const have vanishing Riemann curvature. However, the four-dimensional Riemann curvature tensor has the nonvanishing components

$$
\begin{aligned}
& R^{\alpha}{ }_{t \beta t}=-\frac{\ddot{a}}{a} \delta_{\beta}^{\alpha}=\left(-\dot{H}+H^{2}\right) \delta_{\beta}^{\alpha}, \\
& R^{1}{ }_{212}=R^{1}{ }_{313}=R^{2}{ }_{323}=\dot{a}^{2}
\end{aligned}
$$

and the corresponding components arising from symmetry. Here $\alpha, \beta=1,2,3$. Remarkably, all components which diverge at the singularity are of the type $R_{\text {tata }}$ [29]. Therefore, the singularity arises in the mixed spatiotemporal components.

The geodesic deviation equation along the integral curves of $u=\partial / \partial t$ (which are geodesics with affine parameter $t$ ) is

$$
\dot{u}^{a}=-R^{a}{ }_{c b d} \eta^{b} u^{c} u^{d},
$$

where $\eta^{b}$ is the deviation vector separating neighboring geodesics, chosen to satisfy $\eta^{b} u_{b}=0$. For a Friedmann universe it becomes

$$
\dot{u}^{a}=-R^{a}{ }_{t b t} \eta^{b} \propto \ddot{a},
$$

which at the singularity diverges as $-\infty$. Therefore, when approaching the singularity, the tidal forces manifest themselves as an infinite braking force stopping the further increase of the separation of geodesics but not the evolution along the geodesics. With $\ddot{a}<0$ in the vicinity of the singularity, once the geodesics have passed through, they will approach each other. Therefore a contraction phase will follow: everything that has reached the singularity will bounce back.

\section{The type of the singularity}

In this subsection we shall present the classification of singularities, based on the point of view of finite size objects, which approach these singularities. In principle, finite size objects could be destroyed while passing through the singularity due to the occurring infinite tidal forces. A strong curvature singularity is defined by the requirement that an extended finite object is crushed to zero volume by tidal forces. We give below Tipler's [30] and Królak's [36] definitions of strong curvature singularities together with the relative necessary and sufficient conditions. An alternative definition of the softness of a singularity, based on a Raychaudhuri averaging, was developed by Dabrowski [37].

According to Tipler's definition if every volume element defined by three linearly independent, vorticity-free, geodesic deviation vectors along every causal geodesic through a point $p$ vanishes, a strong curvature singularity is encountered at the respective point $p[26,30]$. The necessary and sufficient condition for a causal geodesic to run into a strong singularity at $\lambda_{s}$ ( $\lambda$ is affine parameter of the curve) [38] is that the double integral

$$
\int_{0}^{\lambda} d \lambda^{\prime} \int_{0}^{\lambda^{\prime}} d \lambda^{\prime \prime}\left|R_{a j b}^{i} u^{a} u^{b}\right|
$$

diverges as $\lambda \rightarrow \lambda_{s}$. A similar condition is valid for lightlike geodesics, with $R_{a j b}^{i} u^{a} u^{b}$ replacing $R_{a b} u^{a} u^{b}$ in the double integral.

Królak's definition is less restrictive. A future-endless, future-incomplete null (timelike) geodesic $\gamma$ is said to terminate in the future at a strong curvature singularity if, for each point $p \in \gamma$, the expansion of every futuredirected congruence of null (timelike) geodesics emanating from $p$ and containing $\gamma$ becomes negative somewhere on $\gamma[36,39]$. The necessary and sufficient condition for a causal geodesic to run into a strong singularity at $\lambda_{s}[38]$ is that the integral

$$
\int_{0}^{\lambda} d \lambda^{\prime}\left|R_{a j b}^{i} u^{a} u^{b}\right|
$$

diverges as $\lambda \rightarrow \lambda_{s}$. Again, a similar condition is valid for lightlike geodesics, with $R_{a j b}^{i} u^{a} u^{b}$ replacing $R_{a b} u^{a} u^{b}$ in the integral.

In flat Friedmann spacetime the comoving observers move on geodesics having four velocity $u=\partial / \partial t$, where $t$ is affine parameter. The nonvanishing components of Riemann tensor are given by Eq. (10). Since $H$ is finite along the geodesics, neither of the integrals (13) and (14) diverge at the singularity. The singularity is weak (soft) according to both Tipler's and Królak's definitions. That means that although the tidal forces become infinite, the finite objects are not necessarily crushed when reaching the singularity (see also Ref. [26]). 


\section{THE PARADOX OF THE SOFT SINGULARITY CROSSING IN THE COSMOLOGICAL MODEL BASED ON THE ANTI-CHAPLYGIN GAS AND DUST UNIVERSE}

We discuss a universe filled with two components. One is the anti-Chaplygin gas with the equation of state (1) and (2) the other is the pressureless dust.

The solution of the continuity equation for the antiChaplygin gas gives the following dependence of its energy density on the scale factor:

$$
\rho_{\mathrm{ACh}}=\sqrt{\frac{B}{a^{6}}-A},
$$

where $B$ is a positive constant, determining the initial condition. The energy density of the dustlike matter is as usual

$$
\rho_{m}=\frac{\rho_{m, 0}}{a^{3}},
$$

where $\rho_{m, 0}$ is a constant.

It is clear that during the expansion of the universe, its scale factor approaches the value

$$
a_{S}=\left(\frac{B}{A}\right)^{\frac{1}{6}},
$$

the energy density of the anti-Chaplygin gas vanishes, and its pressure grows to infinity. That means that the deceleration also becomes infinite. However, the energy density of dust remains finite, hence the same is true also for the Hubble parameter. It is here that the paradox arises: if the universe continues to expand, the expression under the sign of the square root in Eq. (15) becomes negative and the energy density of the anti-Chaplygin gas becomes illdefined. A way out of this situation is only by assuming that at this moment the Hubble parameter changes its sign, while keeping its absolute value (such that the energy density will not have a jump, as implied by the Friedmann equation). This possibility will be studied in detail in the following subsections.

\section{A. Evolutions in the vicinity of the singularity}

Let us substitute Eqs. (15) and (16) into the first Friedmann equation. We shall find its solution for the universe approaching to the soft singularity point at the moment $t_{S}$ (the latter cannot be found analytically, but its value is not important for our analysis),

$$
a(t)=a_{S}-\sqrt{\frac{\rho_{m, 0}}{a_{S}}}\left(t_{S}-t\right)-\sqrt{\frac{2 A a_{S}^{2}}{3 H_{S}}}\left(t_{S}-t\right)^{3 / 2},
$$

where

$$
H_{S}=\sqrt{\frac{\rho_{m, 0}}{a_{S}^{3}}}
$$

is the value of the Hubble parameter at $t=t_{S}$. Correspondingly the leading terms of the energy densities of the anti-Chaplygin gas and dust, also of the pressure of the anti-Chaplygin gas are

$$
\begin{gathered}
\rho_{m}=H_{S}^{2}+3 H_{S}^{3}\left(t_{S}-t\right), \\
\rho_{\mathrm{ACh}}=\sqrt{6 A H_{S}\left(t_{S}-t\right)}, \\
p_{\mathrm{ACh}}=\sqrt{\frac{A}{6 H_{S}\left(t_{S}-t\right)} .}
\end{gathered}
$$

One can see that Eqs. (18), (21), and (22) cannot be continued for $t>t_{S}$ due to the emerging negative quantities under the square roots. The assumption of a sharp transition from expansion to contraction implies the following changes in Eqs. (18)-(22):

$$
\begin{gathered}
a(t)=a_{S}-\sqrt{\frac{\rho_{m, 0}}{a_{S}}}\left|t_{S}-t\right|-\sqrt{\frac{2 A a_{S}^{2}}{3 H_{S}}}\left|t_{S}-t\right|^{3 / 2}, \\
\rho_{m}=H_{S}^{2}+3 H_{S}^{3}\left|t_{S}-t\right|, \\
\rho_{\mathrm{ACh}}=\sqrt{6 A H_{S}\left|t_{S}-t\right|} \\
p_{\mathrm{ACh}}=\sqrt{\frac{A}{6 H_{S}\left|t_{S}-t\right|}} .
\end{gathered}
$$

The quantities (23)-(25) are well-defined and continuous at the moment of the singularity crossing. The expression for the pressure (26) is divergent, but this divergence is integrable and this is sufficient for our purposes. These new expressions satisfy the Friedmann equation, the continuity equations and the equation of state for the anti-Chaplygin gas. However, the time derivatives of these quantities are not continuous and it is the reason of the failure of the Raychaudhuri equation. We shall analyze this problem in the following section, but first we discuss the geodesics in the vicinity of the singularity.

\section{B. Singularity crossing geodesics}

We can integrate explicitly the geodesics Eqs. (8) and (9) in the vicinity of singularity, using Eq. (23) for the cosmological factor, also taken in the vicinity of singularity. Choosing the affine parameter in such a way that the point $\lambda=0$ corresponds to the singularity crossing we obtain up to the second-order in $\lambda$-terms

$$
t=t_{S}+\sqrt{\epsilon+\frac{P^{2}}{a_{S}^{2}}} \lambda+\frac{P^{2} H_{S}}{2 a_{S}^{2}} \operatorname{sgn}(\lambda) \lambda^{2},
$$




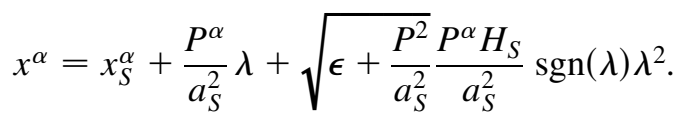

One can see from Eqs. (27) and (28) that not only the time and spatial coordinates of the geodesics are continuous at the soft singularity crossing but also their first derivatives with respect to the affine parameter $\lambda$.

\section{SINGULARITY CROSSING, THE RAYCHAUDHURI EQUATION AND DISTRIBUTIONS}

Let us discuss the expressions for the Hubble parameter and its time derivative in the vicinity of the singularity. Beginning with Eq. (23) we obtain

$$
\begin{gathered}
H(t)=H_{S} \operatorname{sgn}\left(t_{S}-t\right)+\sqrt{\frac{3 A}{2 H_{S} a_{S}^{4}}} \operatorname{sgn}\left(t_{S}-t\right) \sqrt{\left|t_{S}-t\right|}, \\
\dot{H}=-2 H_{S} \delta\left(t_{S}-t\right)-\sqrt{\frac{3 A}{8 H_{S} a_{S}^{4}}} \frac{\operatorname{sgn}\left(t_{S}-t\right)}{\sqrt{\left|t_{S}-t\right|}} .
\end{gathered}
$$

Naturally, the $\delta$-term in $\dot{H}$ arises because of the jump in $H$, as the expansion of the universe is followed by a contraction. To restore the validity of the Raychaudhuri equation we shall add a singular $\delta$-term to the pressure of the antiChaplygin gas, which will acquire the form

$$
p_{\mathrm{ACh}}=\sqrt{\frac{A}{6 H_{S}\left|t_{S}-t\right|}}+\frac{4}{3} H_{S} \delta\left(t_{S}-t\right) .
$$

The equation of state (1) of the anti-Chaplygin gas is preserved, if we also modify the expression for its energy density,

$$
\rho_{\mathrm{ACh}}=\frac{A}{\sqrt{\frac{A}{6 H_{S}\left|t_{S}-t\right|}}+\frac{4}{3} H_{S} \delta\left(t_{S}-t\right)} .
$$

The last expression should be understood in the sense of the composition of distributions (see Appendix A, and the references therein).

In order to prove that $p_{\mathrm{ACh}}$ and $\rho_{\mathrm{ACh}}$ represent a selfconsistent solution of the system of cosmological equations, we shall use the following distributional identities:

$$
\begin{gathered}
{[\operatorname{sgn}(\tau) g(|\tau|)] \delta(\tau)=0,} \\
{[f(\tau)+C \delta(\tau)]^{-1}=f^{-1}(\tau),} \\
\frac{d}{d \tau}[f(\tau)+C \delta(\tau)]^{-1}=\frac{d}{d \tau} f^{-1}(\tau) .
\end{gathered}
$$

Here $g(|\tau|)$ is bounded on every finite interval, $f(\tau)>0$ and $C>0$ is a constant. These identities follow from the propositions 1, 2 and the corollary enounced and proved in Appendix A. The parameter $\tau$ stays instead of the difference $t_{S}-t$.
Because of Eq. (34), the energy density (32) behaves as a continuous function which vanishes at the singularity. Therefore the addition of a Dirac delta term, which is not changing the value of $p_{\mathrm{ACh}}$ at any $\tau \neq 0$ (i.e., $t \neq t_{S}$ ) does not look too drastic and might be considered as some kind of renormalization.

To prove that Friedmann, Raychaudhuri and continuity equations are satisfied we must only investigate those terms, appearing in the field equations, which contain Dirac $\delta$-functions, since without them these equations can be reduced to those we found in the previous section. First, we check the continuity equation for the antiChaplygin gas. Because of the identities (34) and (35), the $\delta(\tau)$-terms occurring in $\rho_{\mathrm{ACh}}$ and $\dot{\rho}_{\mathrm{ACh}}$ could be dropped. We keep them however in order to have the equation of state explicitly satisfied. Then the $\delta(\tau)$-term appearing in $3 H p_{\mathrm{ACh}}$ vanishes, because the Hubble parameter changes sign at the singularity [see Eq. (33)].

The $\delta(\tau)$-term appearing in $\rho_{\mathrm{ACh}}$ does not affect the Friedmann equation due to the identity (34). Finally, the $\delta$-term arising in the time derivative of the Hubble parameter in the left-hand side of the Raychaudhuri equation is compensated by the conveniently chosen $\delta$-term in the right-hand side of Eq. (31).

\section{THE JUNCTION CONDITIONS ACROSS THE SINGULARITY}

In this section we discuss the singularity crossing in a slightly different way, by analyzing the junction conditions. We have to match two spacetime regions across the spacelike hypersurface $\tau=0$. The junction of two spacetime regions has to obey the Israel matching conditions [40], namely, the induced metric should be continuous and the extrinsic curvature of the junction hypersurface could possibly have a jump, which is related to the distributional energy-momentum tensor on the hypersurface by the Lanczos equation. The scale factor being continuous across the singularity the first Israel condition is obeyed. We will next prove that the second Israel junction condition (the Lanczos equations [40,41]) is also satisfied.

For this we have to check whether equation

$$
\frac{\partial}{\partial t}\left(H a^{2}\right)=\left\{-H^{2}+\frac{3}{2}[\tilde{\rho}-\tilde{p}-\bar{p} \delta(\tau)]\right\} a^{2},
$$

(see Appendix C), still implies the Lanczos equation

$$
\left.\Delta H\right|_{t_{s}}=-\frac{3}{2} \bar{p},
$$

derived in Appendix $\mathrm{C}$, when $\tilde{p}+\bar{p} \delta(\tau)=p_{\mathrm{ACh}}, \tilde{\rho}=$ $\rho_{m}+\rho_{\mathrm{ACh}}$ and $\rho_{\mathrm{ACh}}$ is generalized to a distribution

$$
\rho_{\mathrm{ACh}} a^{2}=\frac{P(\tau)}{[R(\tau)+Q(\tau) \delta(\tau)]^{\omega}} .
$$

Here $\omega>0, R(\tau)>0, Q(\tau)>0$ and $P(\tau)$ is bounded. 
When Eq. (36) is applied to a test function $\varphi(\tau)$, the terms containing $H^{2}$ and $\rho_{m}$ give regular contributions and the limits of the respective integrals vanish, similarly as discussed in Appendix C. Also, due to proposition 2 given in Appendix A, the integral of the distributional term containing $\rho_{\mathrm{ACh}}$ becomes

$$
\int_{-\varepsilon}^{\varepsilon} \frac{P(\tau) \varphi(\tau)}{R^{\omega}(\tau)} d \tau
$$

which also vanishes for $\varepsilon \rightarrow 0$. We still have to consider the contributions

$$
\int_{-\varepsilon}^{\varepsilon}[\tilde{p}+\bar{p} \delta(\tau)] \varphi(\tau) a^{2} d \tau .
$$

Although the contribution $\tilde{p} \varphi(\tau) a^{2}$ to the integrand is singular at $\tau=0$, its integral can be conveniently evaluated by the Residue theorem. For this we remark that the integrand is an analytically extendible function into the complex plane in the vicinity of $\tau=0$ and its residue is zero, therefore the integral vanishes. Finally, the contribution containing $\bar{p}$ leads by integration and the limiting process to the right-hand side of the Lanczos equation (37).

Therefore we have proven that the spacetime regions separated by the singular spatial hypersurface, representing the pressure singularity, can be joined. In other words, the singularity becomes traversable.

\section{CONCLUDING REMARKS}

It is known that certain models of cosmological fluids in Friedmann universes, like the anti-Chaplygin gas or the tachyon field with a special potential [32], evolve into a sudden future singularity, which in spite of a diverging pressure, is weak. It was argued that singularities of this kind could be traversable despite infinite tidal forces emerging at the singularity for an infinitesimally short time [26]. In Ref. [29] the process of crossing of the big brake singularity was described in some detail for the tachyon model [32]. (The particularity of the big brake singularity, consists in the fact that at the crossing of such a singularity the Hubble variable is not only finite but vanishes.) We also note recent discussions [42] on crossing the "traditional" big bang and big crunch singularities.

In the present paper we considered a simple cosmological model containing a mixture of anti-Chaplygin gas and dust. We have shown that the geodesics equations and their solutions are still well-defined in this case, however the inclusion of dust generates a nonzero value of the Hubble parameter at the singularity encounter, generating the following paradox. The dust would require a continued expansion, which would make the energy density and pressure of the anti-Chaplygin gas ill-defined. A contraction in turn, would be compatible with the antiChaplygin gas, nevertheless implying an abrupt change of the Hubble parameter from expansion to contraction. The jump in the Hubble parameter implies the appearance of the $\delta$-function in the Raychaudhuri equation (which contains $\dot{H}$ ).

We have cured this situation by redefining the pressure and energy density of the anti-Chaplygin gas as distributions. As an equivalent interpretation, the pressure can be generalized by the addition of a distributional contribution, while the energy density left unchanged, at the price of redefining the equation of state of the anti-Chaplygin gas in a distributional sense. Then all cosmological equations are satisfied in the same distributional sense. We have also shown, that the Israel junction conditions are obeyed through the singular spatial hypersurface, in particular, we have enounced and proved a more general form of the Lanczos equation. The results rely on two propositions and a corollary proven in Appendix A.

The resolution of the paradox at the soft singularity crossing by the introduction of distributional quantities and equations may look unusual, however distributional quantities, localized on hypersurfaces are quite commonly used in general relativity and other gravitational theories. Spacetime regions are frequently matched by the inclusion of distributional layers; also shock-waves can be modeled by Dirac $\delta$-functions. Braneworld models [43,44] arise due to the orbifold boundary conditions, the nonsmoothness of the five-dimensional metric at the brane (the jump in its extrinsic curvature) being directly related to the distributional $3+1$ standard-model fields embedded in the 5-dimensional spacetime. Besides, metrics allowing distributional curvature were considered earlier for studying strings and other distributional sources in general relativity [45]. The applications of the distributional quantities to the study of Schwarzschild geometry and point massive particles in general relativity were used in Refs. [46,47], respectively.

More generically the connection between singularities and the distributional treatment of the physical quantities is well-known in quantum field theory. Indeed, the appearance of the ultraviolet divergences can be understood as the result of the indefiniteness of the product of distributions and the renormalization procedure could be interpreted as a definition of such a product [48].

We hope that the investigations presented here may turn useful in deriving similar results in connection with the traversability of other types of sudden singularities.

While mathematically self-consistent, the scenario presented in this paper may look somewhat counter-intuitive from the physical point of view. This is because its essential ingredient is the abrupt change of the expansion into a contraction. However, such a behavior is not more counterintuitive that the absolutely elastic bounce of the ball from a rigid wall, as known in classical mechanics. Indeed, in the latter case the velocity and the momentum of the ball change their direction abruptly. That means that an infinite force acts from the wall onto the ball during an infinitely small interval of time. The result of this action is however 
integrable and results in a finite change of the momentum of the ball. In fact, the absolutely elastic bounce is an idealization of a process of finite time-span during which inelastic deformations of both the ball and the wall are likely. It is reasonable to think that something similar occurs also in the two-fluid universe model presented in this paper, which undergoes a transition from an expanding to a contracting phase. The smoothing of this process should involve some (temporary) geometrically induced change of the equation of state of matter. Note, that such changes are not uncommon in cosmology. In the tachyon model [32] which was the starting point of our studies of the big brake singularities, there was the tachyonpseudotachyon transformation driven by the continuity of the cosmological evolution. In a cosmological model with the phantom field with the cusped potential [49], the transformations between phantom and standard scalar field were considered. Thus, one can imagine that the real process of the transition from the expansion to contraction induced by passing through a soft singularity can imply some temporary change of the equation of state which makes the above processes smoother. We hope to explore such a scenario in the future.

\section{ACKNOWLEDGMENTS}

We are grateful to V. Gorini, M. O. Katanaev, V.N. Lukash, U. Moschella, D. Polarski and A. A. Starobinsky for useful discussions. The work of Z. K. was supported by OTKA grant No. 100216 and A. K. was partially supported by the RFBR Grant No. 11-02-00643.

\section{APPENDIX A: PROPOSITIONS ON THE PRODUCT AND THE COMPOSITION OF DISTRIBUTIONS}

To investigate how the Friedmann universe crosses a soft singularity, we must solve the field equation in distributional sense. For this purpose we give the definitions of the product and of the composition of distributions and prove two propositions. Fisher derived the following result: $\left[\operatorname{sgn}(\tau)|\tau|^{\lambda}\right] \delta(\tau)=0$ for $\lambda>-1$ [50]. Our first proposition generalizes this equation for $\lambda \geq 0$. The second proposition generalizes Antosik's result: $[1+\delta(\tau)]^{-1}=1$ [51]. Finally, we show a corollary.

Let $\rho(\tau)$ be any infinitely differentiable function having the following properties: (i) $\rho(\tau)=0$ for $|\tau| \geq 1$; (ii) $\rho(\tau) \geq 0$; (iii) $\rho(\tau)=\rho(-\tau)$; (iv) $\int_{-1}^{1} \rho(\tau) d \tau=1$. Then $\delta_{n}(\tau)=n \rho(n \tau)$ (with $n=1,2, \ldots$ ) is a regular sequence of infinitely differentiable functions converging to Dirac delta function: $\lim _{n \rightarrow \infty}\left\langle\delta_{n}, \varphi\right\rangle=\langle\delta, \varphi\rangle$ for any $\varphi \in$ $\mathcal{D}$ [52]. Here $\mathcal{D}$ denotes the space of test functions having continuous derivatives of all orders and compact support. The action of an $f \in \mathcal{D}^{\prime}$ distribution on test functions $\varphi$ is given by $\langle f, \varphi\rangle$, which in the case when $f$ is an ordinary locally summable function is nothing but $\int_{-\infty}^{\infty} f(\tau) \varphi(\tau) d \tau$.
We note that $\delta_{n}(\tau)$ has the compact support $(-1 / n, 1 / n)$. We will also use the $n$-th derivative of $f \in \mathcal{D}^{\prime}$ acts as $\left\langle d f(\tau) / d \tau^{n}, \varphi\right\rangle=(-1)^{n}\left\langle f(\tau), d \varphi / d \tau^{n}\right\rangle$.

For an arbitrary distribution $f$, the function $f_{n}(\tau)=$ $f * \delta_{n} \equiv\left\langle f(\tau-x), \delta_{n}(x)\right\rangle$ gives a sequence of infinitely differentiable functions converging to $f$.

Definition 1. The commutative product of $f$ and $g$ exists and is equal to $h$ on the open interval $(a, b)$ $(-\infty \leq a<b \leq \infty)$ if

$$
\lim _{n \rightarrow \infty}\left\langle f_{n} g_{n}, \varphi\right\rangle=\langle h, \varphi\rangle
$$

for any $\varphi \in \mathcal{D}$ with support contained in the interval $(a, b)$ [52]. ${ }^{1}$

Proposition 1. The commutative product of $\operatorname{sgn}(\tau) g(|\tau|)$ and $\delta(\tau)$ exists and

$$
[\operatorname{sgn}(\tau) g(|\tau|)] \delta(\tau)=0
$$

for arbitrary $g(|\tau|)$ bounded on every finite interval.

Proof. We would like to show that $\langle[\operatorname{sgn}(\tau) g(|\tau|)] \delta(\tau), \varphi\rangle=0$. Using the mean value theorem $\varphi(\tau)=\varphi(0)+\tau d \varphi(\xi \tau) / d \tau$ with $0 \leq \xi \leq 1$, we have

$$
\begin{aligned}
& \left|\left\langle[\operatorname{sgn}(\tau) g(|\tau|)]_{n} \delta_{n}(\tau), \varphi\right\rangle\right| \\
& \leq\left|\varphi(0) \int_{-1 / n}^{1 / n}[\operatorname{sgn}(\tau) g(|\tau|)]_{n} \delta_{n}(\tau) d \tau\right| \\
& \quad+\sup _{|\tau| \leq 1 / n}\left|\frac{d \varphi(\tau)}{d \tau}\right| \int_{-1 / n}^{1 / n}\left|\tau[\operatorname{sgn}(\tau) g(|\tau|)]_{n} \delta_{n}(\tau)\right| d \tau .
\end{aligned}
$$

The first integral on the right side of the above equation vanishes because the integrand is an odd function. For the second integrand, we have

$$
\begin{aligned}
& \int_{-1 / n}^{1 / n}\left|\tau[\operatorname{sgn}(\tau) g(|\tau|)]_{n} \delta_{n}(\tau)\right| d \tau \\
& \quad=\int_{-1 / n}^{1 / n}\left|\tau \delta_{n}(\tau)\right| \int_{-1 / n}^{1 / n}|g(|\tau-x|)| \delta_{n}(x) d x d \tau \\
& \quad \leq n \sup _{|\tau| \leq 1 / n}|\rho(\tau)| \int_{-1 / n}^{1 / n}\left|\tau \delta_{n}(\tau)\right| \int_{-1 / n}^{1 / n}|g(|\tau-x|)| d x d \tau \\
& \quad \leq 2 \sup _{|\tau| \leq 1 / n}|\rho(\tau)| \sup _{|\tau| \leq 1 / n}|g(|\tau|)| \int_{-1 / n}^{1 / n}\left|\tau \delta_{n}(\tau)\right| d \tau \\
& \quad=\frac{2}{n} \sup _{|\tau| \leq 1 / n}|\rho(\tau)| \sup _{|\tau| \leq 1 / n}|g(|\tau|)| \int_{-1}^{1}|y \rho(y)| d y \\
& \quad \leq \frac{2}{n} \sup _{|\tau| \leq 1 / n}|\rho(\tau)| \sup _{|\tau| \leq 1 / n}|g(|\tau|)|,
\end{aligned}
$$

that vanishes for $n \rightarrow \infty$.

Definition 2. The composition $F(f)$ of distributions $F$ and $f$ exists and is equal to $h \in \mathcal{D}^{\prime}$ on the interval $(a, b)$ if

\footnotetext{
${ }^{1}$ This definition can be generalized for the cases when the usual limit does not exist by taking the so-called neutrix limit $[52,53]$. However, we do not need this more general definition here.
} 


$$
\lim _{n \rightarrow \infty}\left[\lim _{m \rightarrow \infty} \int_{a}^{b} F_{n}\left(f_{m}(\tau)\right) \varphi(\tau) d \tau\right]=\langle h, \varphi\rangle
$$

for all $\varphi \in \mathcal{D}$ with support contained in the interval $(a, b)^{2}$

Proposition 2. The composition of distribution $P(\tau)[R(\tau)+Q(\tau) \delta(\tau)]^{-\omega}$ (where $\omega>0, P(\tau)$ is bounded, $R(\tau) \neq 0$, and in some range close $\tau=0$ the signs of $R(\tau)$ and $Q(\tau)$ are the same if $Q(\tau) \neq 0$ ) exists if $P(\tau) / R^{\omega}(\tau)$ exists $^{3}$ and

$$
\frac{P(\tau)}{[R(\tau)+Q(\tau) \delta(\tau)]^{\omega}}=\frac{P(\tau)}{R^{\omega}(\tau)}
$$

Proof. By the definition of composition of distributions, we should calculate

$$
\begin{aligned}
& \left\langle\frac{P_{n}(\tau)}{\left[R_{m}(\tau)+Q_{m}(\tau) \delta_{m}(\tau)\right]_{n}^{\omega}}, \varphi(\tau)\right\rangle \\
& \quad=\int_{-\infty}^{\infty} \int_{-1 / n}^{1 / n} \frac{\varphi(\tau) P_{n}(\tau) \delta_{n}(x) d x d \tau}{\left[R_{m}(\tau-x)+Q_{m}(\tau) \delta_{m}(\tau-x)\right]^{\omega}} .
\end{aligned}
$$

Performing a change of the variables as $\tau=\tau, y=$ $m(\tau-x)$, we have

$$
\begin{aligned}
= & -\frac{1}{m} \int_{-\infty}^{\infty} \int_{-\infty}^{\infty} \frac{\varphi(\tau) P_{n}(\tau) \delta_{n}(\tau-y / m)}{\left[R_{m}(y / m)+m Q_{m}(y / m) \rho(y)\right]^{\omega}} d y d \tau \\
= & -\frac{1}{m} \int_{-\infty}^{\infty} \int_{\Omega_{1}} \frac{\varphi(\tau) P_{n}(\tau) \delta_{n}(\tau-y / m)}{R_{m}^{\omega}(y / m)} d y d \tau \\
& -\frac{1}{m} \int_{-\infty}^{\infty} \int_{\Omega_{2}} \frac{\varphi(\tau) P_{n}(\tau) \delta_{n}(\tau-y / m)}{\left[R_{m}(y / m)+m Q_{m}(y / m) \rho(y)\right]^{\omega}} d y d \tau,
\end{aligned}
$$

where $\Omega_{2}=\{y:|y|<1$ and $\rho(y) \neq 0\}$ and $\Omega_{1}=\mathbb{R}-\Omega_{2}$. The double limit of the first term is

$$
\begin{aligned}
& \lim _{n \rightarrow \infty} \lim _{m \rightarrow \rightarrow \infty}-\frac{1}{m} \int_{-\infty}^{\infty} d \tau \varphi(\tau) P_{n}(\tau) \int_{\Omega_{1}} \frac{\delta_{n}(\tau-y / m)}{R_{m}^{\omega}(y / m)} d y \\
& =\lim _{n \rightarrow \infty} \lim _{m \rightarrow \rightarrow \infty} \int_{-\infty}^{\infty} d \tau \varphi(\tau) P_{n}(\tau) \int_{\substack{|x|<1 / n, \Omega_{1} \\
m|\tau-x| \in \Omega_{1}}} \frac{\delta_{n}(x)}{R_{m}^{\omega}(\tau-x)} d x \\
& =\left\langle\frac{P(\tau)}{R^{\omega}(\tau)}, \varphi(\tau)\right\rangle .
\end{aligned}
$$

We investigate the absolute value of the second integral. According to our assumptions for $R$ and $Q$, and since we are interested in $m \rightarrow \infty$, we can choose $m$ large enough to let the signs of $R$ and $Q$ be the same, then for $\omega>0$

\footnotetext{
${ }^{2}$ This definition can be generalized for the cases when the usual limit does not exist by taking double neutrix limit [54-56].

${ }^{3}$ We note that this proposition can be held even if $P(\tau)=1$ and $R(\tau)=\delta(\tau)$ with $\omega=1,2, \ldots$ Indeed, $\delta^{-\omega}(\tau)$ exists in neutrix limit and $\delta^{-\omega}(\tau)=0$ [55]. Thus in definition 2, the usual limit must be changed for neutrix limit for this case.
}

$$
\begin{aligned}
& \left|\frac{1}{m} \int_{-\infty}^{\infty} \int_{\Omega_{2}} \frac{\varphi(\tau) P_{n}(\tau) \delta_{n}(\tau-y / m)}{\left[R_{m}(y / m)+m Q_{m}(y / m) \rho(y)\right]^{\omega}} d y d \tau\right| \\
& \quad \leq\left|\frac{1}{m^{1+\omega}} \int_{-\infty}^{\infty} \varphi(\tau) P_{n}(\tau) \int_{\Omega_{2}} \frac{\delta_{n}(\tau-y / m)}{Q_{m}(y / m) \rho^{\omega}(y)} d y d \tau\right| .
\end{aligned}
$$

Performing a change of the variables as $z=n(\tau-y / m)$, $y=y$, we have

$$
\begin{aligned}
\leq & \frac{1}{m^{1+\omega}} \int_{-1}^{1} \int_{\Omega_{2}}\left|\varphi\left(\frac{z}{n}+\frac{y}{m}\right) P_{n}\left(\frac{z}{n}+\frac{y}{m}\right) \frac{\rho(z)}{\rho^{\omega}(y)}\right| d y d z \\
\leq & \frac{1}{m^{1+\omega}} \sup _{\Omega_{2},|z| \leq 1} \mid \varphi\left(\frac{z}{n}+\frac{y}{m}\right) P_{n}\left(\frac{z}{n}+\frac{y}{m}\right) \rho^{-\omega}(y) \\
& \times \mid \int_{-1}^{1} \rho(z) d z \int_{-1}^{1} d y \\
= & \frac{2}{m^{1+\omega}} \sup _{\Omega_{2},|z| \leq 1}\left|\varphi\left(\frac{z}{n}+\frac{y}{m}\right) P_{n}\left(\frac{z}{n}+\frac{y}{m}\right) \rho^{-\omega}(y)\right|,
\end{aligned}
$$

that vanishes for $m \rightarrow \infty$ if $P$ is bounded.

Corollary 1. The distribution $d\{P(\tau)[R(\tau)+$ $\left.Q(\tau) \delta(\tau)]^{-\omega}\right\} / d \tau$ (with the same properties for $P, R, Q$ and $\omega$ as in proposition (2) exists if $P(\tau) / R^{\omega}(\tau)$ and its derivative exist, and

$$
\frac{d}{d \tau} \frac{P(\tau)}{[R(\tau)+Q(\tau) \delta(\tau)]^{\omega}}=\frac{d}{d \tau} \frac{P(\tau)}{R^{\omega}(\tau)} .
$$

Proof. Applying the derivative of a distribution at tests functions, and using the fact that $d \varphi / d \tau \in \mathcal{D}$ for any $\varphi \in \mathcal{D}$, and by the proposition 2 , we have

$$
\begin{aligned}
\left\langle\frac{d}{d \tau}\right. & \left.\frac{P(\tau)}{[R(\tau)+Q(\tau) \delta(\tau)]^{\omega}}, \varphi\right\rangle \\
& =-\left\langle\frac{P(\tau)}{[R(\tau)+Q(\tau) \delta(\tau)]^{\omega}}, \frac{d}{d \tau} \varphi\right\rangle \\
& =-\left\langle\frac{P(\tau)}{R^{\omega}(\tau)}, \frac{d}{d \tau} \varphi\right\rangle=\left\langle\frac{d}{d \tau} \frac{P(\tau)}{R^{\omega}(\tau)}, \varphi\right\rangle .
\end{aligned}
$$

\section{APPENDIX B: TWO SIMPLE EXAMPLES OF THE PRODUCT AND OF THE DECOMPOSITION OF DISTRIBUTIONS}

The definition of the product and the composition of distributions, used in this paper and presented in Appendix A are not often encountered in physics. Thus, to give the reader some flavor of the corresponding considerations, using simpler means we decided to give two semiheuristic examples of such products and compositions. We consider first a remarkable formula

$$
\mathcal{P}\left(\frac{1}{x}\right) \delta(x)=-\frac{1}{2} \delta^{\prime}(x)
$$

which was first proven in Ref. [57]. Here $\mathcal{P}$ means the principal value of the corresponding function. We shall prove here that the regularizing succession of functions with compact support $\rho$, employed in Appendix A and the 
references therein, can be chosen alternatively as the family of the Cauchy-Lorentz functions

$$
f_{\epsilon}(x)=\frac{1}{\pi} \frac{\epsilon}{x^{2}+\epsilon^{2}} .
$$

It is well known that when the small parameter $\epsilon \rightarrow 0$, the functions of this family tend in the distributional sense to the Dirac $\delta$-function. Obviously, the convolution of the function (B2) with the Dirac $\delta$-function gives again the same function (B2),

$$
f_{\epsilon} * \delta(x)=f_{\epsilon}(x)
$$

The calculation of the convolution of the principal value $\mathcal{P}\left(\frac{1}{x}\right)$ with the function $f_{\epsilon}(x)$ is slightly more complicated,

$$
\begin{aligned}
\mathcal{P}\left(\frac{1}{x}\right) * f_{\epsilon}(x)= & \lim _{\varepsilon \rightarrow 0}\left(\int_{-\infty}^{x-\varepsilon} d y \frac{1}{x-y} \frac{\epsilon}{\pi\left(y^{2}+\epsilon^{2}\right)}\right. \\
& \left.+\int_{x+\varepsilon}^{\infty} d y \frac{1}{x-y} \frac{\epsilon}{\pi\left(y^{2}+\epsilon^{2}\right)}\right) \\
= & \frac{x}{x^{2}+\epsilon^{2}} .
\end{aligned}
$$

The product of Eqs. (B3) and (B4) is

$$
\mathcal{P}\left(\frac{1}{x}\right)_{\epsilon} * \delta_{\epsilon}(x)=\frac{\epsilon x}{\pi\left(x^{2}+\epsilon^{2}\right)^{2}} .
$$

Let us now consider a family of functions,

$$
\frac{d f_{\epsilon}(x)}{d x}=-\frac{2 x \epsilon}{\pi\left(x^{2}+\epsilon^{2}\right)^{2}} .
$$

One can easily prove that if the family of functions (B2) converges in the distributional sense to the Dirac $\delta$-function, the family of their derivatives (B6) converges to the derivative of the delta function. Now, comparing the right-hand sides of Eqs. (B6) and (B5) we see that when $\epsilon \rightarrow 0$ the product in the left-hand side of Eq. (B5) converges in the distributional sense to $-\frac{1}{2} \delta^{\prime}(x)$ and thus the correctness of the equality (B1) is checked.

Now let us discuss the Antosik identity [51],

$$
\frac{1}{1+\delta(x)}=1
$$

Here we have the composition of the distributions $F(g)$, where $F=\frac{1}{g}$ and $g(x)=1+\delta(x)$. Calculating the convolutions of the distributions $F$ and $g$ with the CauchyLorentz functions (B2) we obtain

$$
\begin{gathered}
F_{\sigma}(g)=F * f_{\sigma}(g)=\frac{g}{g^{2}+\sigma^{2}}, \\
g_{\epsilon}(x)=1+\frac{\epsilon}{x^{2}+\epsilon^{2}} .
\end{gathered}
$$

Correspondingly the composition of these functions is

$$
F_{\sigma}\left(g_{\epsilon}\right)=\frac{1+\frac{\epsilon}{\pi\left(x^{2}+\epsilon^{2}\right.}}{\sigma^{2}+\left(1+\frac{\epsilon}{\pi\left(x^{2}+\epsilon^{2}\right.}\right)^{2}}
$$

and it is easy to check that

$$
\lim _{\sigma \rightarrow 0} \lim _{\epsilon \rightarrow 0} F_{\sigma}\left(g_{\epsilon}\right)=1,
$$

confirming the identity (B7).

\section{APPENDIX C: THE LANCZOS EQUATION}

For a generic junction surface the Lanczos equation emerges from the Gauss-Codazzi relations [43,58]. The projected Lie derivative of the extrinsic curvature $K_{a b}$ in the normal direction $n$ to the surface is

$$
h_{a}^{i} h_{b}^{j} \mathcal{L}_{\mathbf{n}} K_{i j}=-3 \epsilon\left(h_{a}^{i} h_{b}^{k} T_{i k}-\frac{h_{a b}}{2} g^{i k} T_{i k}\right)+Z_{a b}
$$

[Eq. (21) of Ref. [43] in the units $8 \pi G / 3=1$ ], with

$$
Z_{a b}=-\epsilon \mathcal{R}_{a b}+2 K_{a c} K_{b}^{c}-g^{i k} K_{i k} K_{a b}+D_{b} \alpha_{a}-\epsilon \alpha_{b} \alpha_{a} .
$$

Here $g_{a b}$ is the spacetime metric, $h_{a b}=g_{a b}-\epsilon n_{a} n_{b}$ $\left(\epsilon=n^{a} n_{a}=\{-1,1\}\right)$ is the induced metric on the junction surface, and $T_{a b}$ is the energy-momentum tensor. The tensor $Z_{a b}$ depends only on geometrical quantities: $\mathcal{R}_{a b}$ and $D$ are the Ricci tensor and covariant derivative induced on the hypersurface, and $\alpha_{a}=n^{c} \nabla_{c} n_{a}$, with $\nabla$ the fourdimensional covariant derivative.

When the energy-momentum tensor is a sum $T_{i k}=$ $\Pi_{i k}+\Upsilon_{i k} \delta(\tau)$ (where $\tau$ is the coordinate adapted to $n$, i.e., $n=t_{S}^{-1} \partial / \partial \tau$, and $n^{a} \Upsilon_{a b}=0$ ), with $\Pi_{i k}$ the regular four-dimensional part and $Y_{i k}$ the distributional part on the hypersurface, integration of Eq. (C1) across $\tau$ through an infinitesimal range containing the hypersurface keeps only the distributional part, leading to the Lanczos equations $[40,41]$

$$
\Delta K_{a b}=-3 \epsilon\left(\Upsilon_{a b}-\frac{\Upsilon}{2} h_{a b}\right),
$$

or equivalently

$$
-3 \epsilon \Upsilon_{a b}=\Delta K_{a b}-h_{a b} \Delta K .
$$

Here $Y$ is the trace of $Y_{a b}$. As $Z_{a b}$ is finite, its contribution to the integral across the infinitesimal range also vanishes. Without a distributional energy-momentum part, the extrinsic curvature should be continuous.

Let us now specialize this for a junction along a maximally symmetric $\tau=0$ spacelike hypersurface (a hyperplane with $\mathcal{R}_{a b}=0$ ) embedded in a flat Friedmann spacetime. The normal vector $n$ has zero acceleration $\alpha_{a}=$ 0 and the extrinsic curvature becomes $K_{a b}=\dot{a} a \tilde{h}_{a b}$, with $\tilde{h}_{a b}$ the three-dimensional Euclidean metric. The curvature 
term is $Z_{a b}=-H^{2} a^{2} \tilde{h}_{a b}$ and the energy-momentum tensors are $\Pi_{a b}=\tilde{\rho} n_{a} n_{b}+\tilde{p} a^{2} \tilde{h}_{a b}$ and $\Upsilon_{a b}=\bar{p} a^{2} \tilde{h}_{a b}$. Since the projected Lie-derivative in Eq. (C1) becomes a time derivative, the equation reads

$$
\frac{\partial}{\partial t}\left(H a^{2}\right)=\left\{-H^{2}+\frac{3}{2}[\tilde{\rho}-\tilde{p}-\bar{p} \delta(\tau)]\right\} a^{2},
$$

which is a combination of the Raychaudhuri and Friedmann equations. For finite $H, \tilde{\rho}$ and $\tilde{p}$ as before the integration of Eq. (C5) across an infinitesimal time range $\tau$ leads to the Lanczos equation

$$
\left.\Delta H\right|_{t_{s}}=-\frac{3}{2} \bar{p}
$$

[1] L. D. Landau and E. M. Lifshitz, The Classical Theory of Fields (Pergamon Press, Oxford, 1975), 4th ed..

[2] C. W. Misner, K. S. Thorne, and J. A. Wheeler, Gravitation (W. H. Freeman, San Francisco, 1973).

[3] S.W. Hawking and G.F. R. Ellis, The Large Scale Structure of Space-time (Cambridge University Press, Cambridge, 1973).

[4] S. W. Hawking and R. Penrose, Proc. R. Soc. A 314, 529 (1970).

[5] R. Penrose, Structure of Space-time (W. A. Benjamin, New York, 1970).

[6] I. M. Khalatnikov and E. M. Lifshitz, Phys. Rev. Lett. 24, 76 (1970); V. A. Belinsky, I. M. Khalatnikov, and E. M. Lifshitz, Adv. Phys. 19, 525 (1970).

[7] C. W. Misner, Phys. Rev. Lett. 22, 1071 (1969).

[8] J. D. Barrow, G. J. Galloway, and F. J. Tipler, Mon. Not. R. Astron. Soc. 223, 835 (1986).

[9] J. D. Barrow, Phys. Lett. B 235, 40 (1990).

[10] Yu. Shtanov and V. Sahni, Classical Quantum Gravity 19, L101 (2002).

[11] P. Tretyakov, A. Toporensky, Yu. Shtanov, and V. Sahni, Classical Quantum Gravity 23, 3259 (2006).

[12] J.D. Barrow, Classical Quantum Gravity 21, L79 (2004).

[13] J. D. Barrow, Classical Quantum Gravity 21, 5619 (2004).

[14] J. D. Barrow, A. B. Batista, J. C. Fabris, and S. Houndjo, Phys. Rev. D 78, 123508 (2008).

[15] M. P. Dabrowski, T. Denkiewicz, and M. A. Hendry, Phys. Rev. D 75, 123524 (2007).

[16] J. D. Barrow and S.Z.W. Lip, Phys. Rev. D 80, 043518 (2009).

[17] J. D. Barrow, A. B. Batista, G. Dito, J.C. Fabris, and S. Houndjo, Phys. Rev. D 84, 123518 (2011).

[18] H. Maeda, Phys. Rev. D 85, 124012 (2012).

[19] A. Riess et al., Astron. J. 116, 1009 (1998); S. J. Perlmutter et al., Astrophys. J. 517, 565 (1999).

[20] V. Sahni and A. A. Starobinsky, Int. J. Mod. Phys. D 9, 373 (2000); 15, 2105 (2006); T. Padmanabhan, Phys. Rep. 380, 235 (2003); P. J. E. Peebles and B. Ratra, Rev. Mod. Phys. 75, 559 (2003); E. J. Copeland, M. Sami, and S. Tsujikawa, Int. J. Mod. Phys. D 15, 1753 (2006).

[21] T. Denkiewicz, M. P. Dabrowski, H. Ghodsi, and M. A. Hendry, Phys. Rev. D 85, 083527 (2012).

[22] Z. Keresztes, L. Á. Gergely, V. Gorini, U. Moschella, and A. Yu. Kamenshchik, Phys. Rev. D 79, 083504 (2009).

[23] J. de Haro, J. Amoros, and E. Elizalde, Phys. Rev. D 85, 123527 (2012).
[24] A. Yu. Kamenshchik, C. Kiefer, and B. Sandhöfer, Phys. Rev. D 76, 064032 (2007).

[25] A. Y. Kamenshchik and S. Manti, Phys. Rev. D 85, 123518 (2012).

[26] L. Fernández-Jambrina and R. Lazkoz, Phys. Rev. D 70, 121503 (2004).

[27] L. Fernández-Jambrina and R. Lazkoz, Phys. Lett. B 670, 254 (2009).

[28] A. Balcerzak and M.P. Dabrowski, Phys. Rev. D 73, 101301 (2006).

[29] Z. Keresztes, L. Á. Gergely, A. Yu. Kamenshchik, V. Gorini, and D. Polarski, Phys. Rev. D 82, 123534 (2010).

[30] F. J. Tipler, Phys. Lett. A64, 8 (1977).

[31] V. N. Lukash, E. V. Mikheeva, and V. N. Strokov, Phys. Usp. 55, 204 (2012).

[32] V. Gorini, A. Yu. Kamenshchik, U. Moschella, and V. Pasquier, Phys. Rev. D 69, 123512 (2004).

[33] B. Carter, Phys. Lett. B 224, 61 (1989); A. Vilenkin, Phys. Rev. D 41, 3038 (1990).

[34] A. Yu. Kamenshchik, U. Moschella, and V. Pasquier, Phys. Lett. B 511, 265 (2001).

[35] J. C. Fabris, S. V. B. Goncalves, and P.E. de Souza, Gen. Relativ. Gravit. 34, 53 (2002); N. Bilic, G. B. Tupper, and R. D. Viollier, Phys. Lett. B 535, 17 (2002); M. C. Bento, O. Bertolami, and A.A. Sen, Phys. Rev. D 66, 043507 (2002); V. Gorini, A. Yu. Kamenshchik, and U. Moschella, Phys. Rev. D 67, 063509 (2003).

[36] A. Królak, Classical Quantum Gravity 3, 267 (1986).

[37] M. P. Dabrowski, Phys. Lett. B 702, 320 (2011).

[38] C. J. S. Clarke and A. Królak, J. Geom. Phys. 2, 127 (1985).

[39] W. Rudnicki, R. J. Budzynski, and W. Kondracki, Mod. Phys. Lett. A 17, 387 (2002).

[40] W. Israel, Nuovo Cimento B 44, 1 (1966); 48, 463(E) (1967).

[41] C. Lanczos, Phys. Z. 23, 539 (1922); Ann. Phys. (Leipzig) 379, 518 (1924).

[42] I. Bars, S. H. Chen, and N. Turok, Phys. Rev. D 84, 083513 (2011); I. Bars, S. H. Chen, P. J. Steinhardt, and N. Turok, arXiv:1112.2470; arXiv:1207.1940.

[43] L. Á. Gergely, Phys. Rev. D 68, 124011 (2003).

[44] L. Randall and R. Sundrum, Phys. Rev. Lett. 83, 4690 (1999); T. Shiromizu, K. I. Maeda, and M. Sasaki, Phys. Rev. D 62, 024012 (2000); Z. Kovács and L. Á. Gergely, Phys. Rev. D 77, 024003 (2008); L. Á. Gergely, Phys. Rev. D 78, 084006 (2008); Z. Keresztes and L. Á. Gergely, Classical Quantum Gravity 27, 105009 (2010); R. 
Maartens and K. Koyama, Living Rev. Relativity 13, 5 (2010).

[45] R. Geroch and J. Traschen, Phys. Rev. D 36, 1017 (1987).

[46] H. Balasin and H. Nachbagauer, Classical Quantum Gravity 10, 2271 (1993).

[47] M. O. Katanaev, arXiv:1207.3481.

[48] N. N. Bogoliubov and D. V. Shirkov, Introduction to the Theory of Quantized Fields (Interscience, New York, 1980), 3rd ed..

[49] A. A. Andrianov, F. Cannata, and A. Y. Kamenshchik, Phys. Rev. D 72, 043531 (2005); F. Cannata and A. Y. Kamenshchik, Int. J. Mod. Phys. D 16, 1683 (2007).

[50] B. Fisher, Proc. Cambridge Philos. Soc. 73, 317 (1973).
[51] P. Antosik, University of Wisconsin Report No. 9, 19881989.

[52] C. L. Zhi and B. Fisher, Proc. R. Soc. A 426, 425 (1989).

[53] J. G. van der Corput, Journal d'analyse mathematique 7, 291 (1959).

[54] B. Fisher, Rostocker Mathematisches Kolloquium 28, 75 (1985).

[55] E. Özçağ, Appl. Math. Lett. 14, 419 (2001).

[56] B. Fisher and A. Kılıçman, J. Appl. Math. Mech. 2011, 1 (2011).

[57] J. Mikusinski, Bull. Acad. Polon. Sci. Sér. Sci. Math. Astr. Phys. 14, 511 (1966).

[58] P. Bowcock, C. Charmousis, and R. Gregory, Classical Quantum Gravity 17, 4745 (2000). 